\title{
Necrotizing Fasciitis of the Abdominal Wall in a Patient with Diverticulitis
}

\author{
${ }^{1}$ Ricardo A Jacquez, ${ }^{2}$ Uwe M Fischer, ${ }^{3}$ Michael D Yaakovian, ${ }^{4}$ Peter A Walker, ${ }^{5}$ Shinil K Shah
}

\begin{abstract}
Intra-abdominal infections, such as diverticulitis causing necrotizing fasciitis of the abdominal wall or extremities, are extremely rare and carry a high mortality rate. We present the case of a 52-year-old man with multiple medical comorbidities and diverticulitis who presented to our service with necrotizing fasciitis of the left lateral and posterior abdominal wall. The relevant literature is discussed.
\end{abstract}

Keywords: Diverticulitis, Necrotizing fasciitis, Abdominal wall.

How to cite this article: Jacquez RA, Fischer UM, Yaakovian MD, Walker PA, Shah SK. Necrotizing Fasciitis of the Abdominal Wall in a Patient with Diverticulitis. J Trauma Crit Care Emerg Surg 2014;3(1):37-38.

Source of support: Nil

Conflict of interest: None

\section{RESUMEN}

Infecciones intra-abdominales, tales como la diverticulitis que causan fascitis necrotizante de la pared abdominal o en las extremidades, son extremadamente raro y llevar a una alta tasa de mortalidad. Se presenta el caso de un hombre de 52 años de edad, con múltiples comorbilidades médicas y diverticulitis que presentó a nuestro servicio con necrotizante La fascitis de la pared abdominal lateral y posterior izquierda. La Se discute la literatura relevante.

Palabras claves: Diverticulitis, Fascitis necrotizante, La pared abdominal.

\section{INTRODUCTION}

Necrotizing fasciitis of the abdominal wall or extremities secondary to intraabdominal pathology is extremely rare.

\footnotetext{
${ }^{1}$ Resident, ${ }^{2}$ Clinical Assistant Professor, ${ }^{3}$ Surgeon

${ }^{4,5}$ Clinical Instructor

${ }^{1}$ Department of Surgery, New York Hospital Queens, New York, USA

2,4,5Department of Surgery, University of Texas Medical School Houston, USA

${ }^{3}$ Department of Surgery, Mid-Atlantic Permanente Medical Group, USA

Corresponding Author: Shinil K Shah, Clinical Instructor Department of Surgery, University of Texas Medical School, Houston, Texas, USA, Phone: 2818416034, e-mail: shinil.k.shah@uth.tmc.edu
}

We present the case of a man with multiple medical comorbidities and diverticulitis who presented to our service with necrotizing fasciitis of the left lateral and posterior abdominal wall.

\section{CASE REPORT}

A 52-year-old African-American man with a history of dilated alcoholic cardiomyopathy, congestive heart failure, hypertension, and anemia arrived to our emergency center with 5 days of progressively worsening left flank pain. The pain was characterized as a constant, sharp $9 / 10$ pain, which radiated through both flanks to the left side of his back. He reported subjective fevers, chills, progressive weakness, difficulty with ambulation, and unexplained bruising to the site of pain 3 days prior to his arrival. He has only remarkable surgical history of upper extremity orthopedic surgery many years prior. His social history was remarkable for tobacco, alcohol and marijuana use. Approximately, 4 years prior to arrival, he had findings of diverticulitis on computed tomography (CT) scan of the abdomen.

On initial evaluation, the patient was febrile (100.9 F) and tachycardic (low 100s) but normotensive. His physical examination was remarkable for left lower quadrant tenderness as well as left flank and back erythema and tenderness. His laboratory evaluation demonstrated leukocytosis (white blood cell count of $24 \mathrm{~K} / \mu \mathrm{l}$ (7 bands, 82 neutrophils)). CT scan of the abdomen (Fig. 1) demonstrated significant inflammatory changes and a large amount of gas $(8.1 \times 5.3$ $\times 4.4 \mathrm{~cm}$ ) within the subcutaneous fat and involving the abdominal wall musculature of the posterior lateral abdominal wall. Extension of the inflammation to include the left retroperitoneum, left pararenal and lateral conal fascia, and around the descending colon was noted. An enterocutaneous fistula could not be excluded. The findings were consistent with a necrotizing soft tissue infection and the patient was emergently taken to the operating room for incision and drainage.

In the operating room, the area of abnormality along the left lateral abdominal wall and flank was explored and found to contain necrotic muscle and fascia with multiple loculated collections of purulent brown fluid. Extensive debridement and drainage was undertaken and the patient was transferred to the intensive care unit (ICU) for postoperative care and broad-spectrum antibiotics. He underwent 


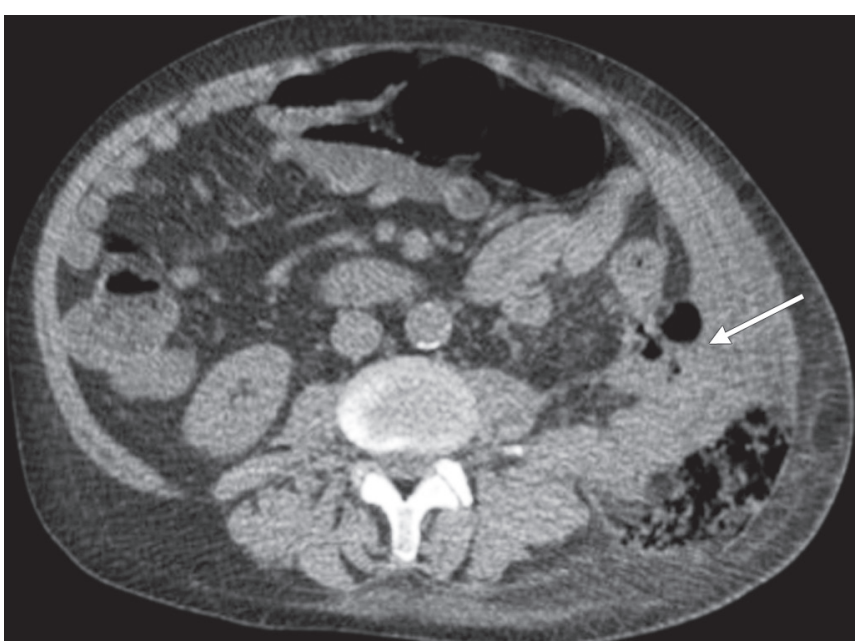

Fig. 1: Computed tomography scan of the abdomen demonstrating extensive inflammatory changes of the posterior and lateral left abdominal wall consistent with a necrotizing soft tissue infection. Additionally, there is extension of inflammation around the descending colon, suspicious for an enterocutaneous fistula (arrow)

a washout the following day with minimal additional necrotic tissue debrided. Secondary to persistent fevers and leukocytosis and the suggestion of an enterocutaneous fistula on initial CT scan, the patient underwent a contrast enema study on hospital day 4. Findings were consistent with diverticulitis but no fistulous tract seen, raising the possibility of a sealed perforation or subclinical fistulous tract (Fig. 2). Operating room cultures demonstrated polymicrobial infection [Streptococcus beta hemolytic, Gamma hemolytic Streptococcus (not Enterococcus) and Prevotella].

Given response to operative debridement and drainage and broad-spectrum antibiotics with no evidence of enterocutaneous fistula, colonic diversion or resection was not necessary. The patient was eventually discharged home with home negative pressure wound therapy. There has been no recurrence of symptoms with approximately 2 years of follow-up.

\section{CONCLUSION}

Necrotizing fasciitis associated with diverticulitis is rarely reported. Galbut et al ${ }^{1}$ reported the first case in the literature of a 71-year-old woman with diverticulitis who presented with left-sided abdominal wall necrotizing fasciitis necessitating extensive debridement. The cause was determined to be a fistula from the sigmoid colon and diversion with a transverse loop colostomy was necessary. The patient died 18 days after initial hospitalization due to generalized sepsis. Since then, there have been approximately 20 reported cases of diverticulitis presenting as a necrotizing soft tissue infection, including some cases of Fournier's gangrene secondary to diverticulitis. ${ }^{2}$

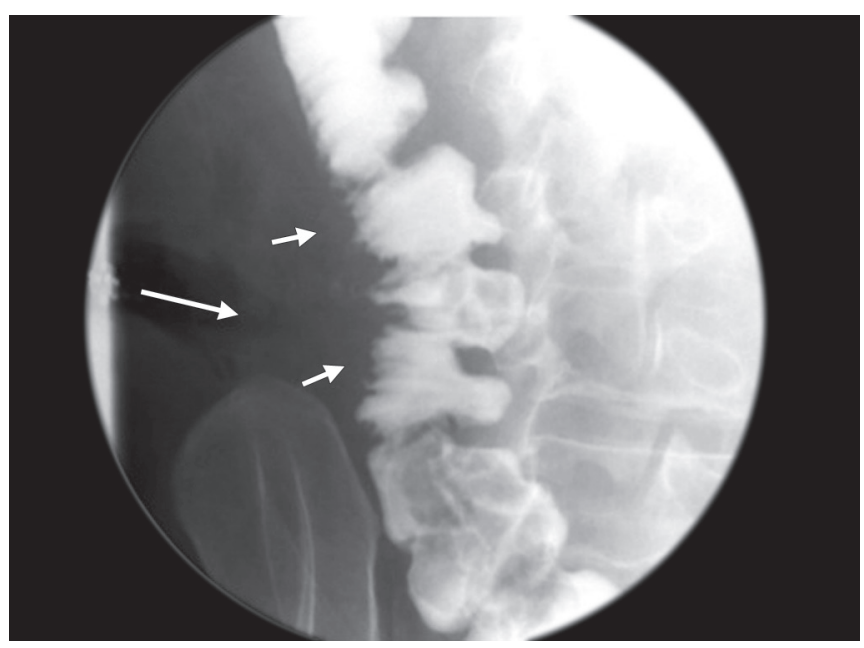

Fig. 2: Contrast enema study of the colon demonstrating diverticulum in the region of the colon seen on CT scan but no fistulous tract seen. The caliber of mid descending colon is smaller than those of other parts of the colon. There is small amount of spasm in the same area and there is slight, asymmetric irregularity and serration of the outline of the colon at the level of the abscess. The changes are consistent with evolving diverticulitis, apparently sealed. Postevacuation films (not shown) also do not show fistulous communication to the flank abscess

In nearly all cases, the etiology is a perforation or fistula from the involved colonic segment. Management of the patients is complex secondary to the aggressive nature of these necrotizing infections. Aggressive control of sepsis, including broad-spectrum antibiotics covering anaerobic, Gram-negative and Gram-positive organisms, and wide and aggressive debridement of infected tissues is imperative. Source control of the colon is important and frequently includes proximal diversion with resection of affected segment if possible. Multiple scheduled operative reexplorations are mandatory to verify continued control of infection. The use of temporary abdominal closure is oftentimes mandatory. In the patient who survives, issues of abdominal wall reconstruction and soft tissue coverage become central to the goals of care.

In the case presented, no persistent fistula from the colon was noted on evaluation with contrast enema. We suspect given the CT findings, operative findings, and culture results demonstrating anaerobic organisms that the etiology of necrotizing fasciitis in this patient was secondary to a contained diverticular perforation.

\section{REFERENCES}

1. Galbut DL, Gerber DL, Belgraier AH. Spontaneous necrotizing fasciitis occurrence secondary to occult diverticulitis. JAMA 1977;238(21):2302.

2. Gerber GS, Guss SP, Pielet RW. Fournier's gangrene secondary to intra-abdominal process. Urology 1994;44(5):779-782. 